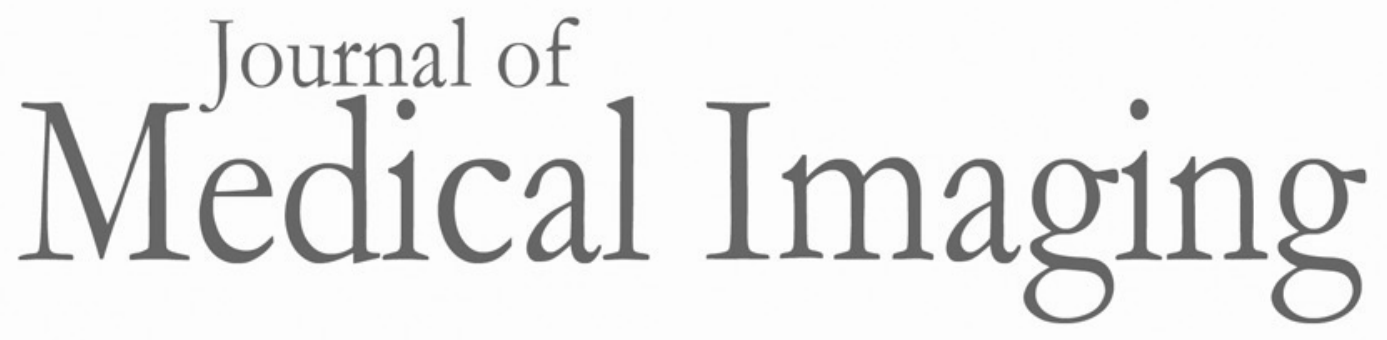

\title{
Recent advances in cardiac computed tomography dose reduction strategies: a review of scientific evidence and technical developments
}

Sandeep S. Hedgire

Vinit Baliyan

Brian B. Ghoshhajra

Mannudeep K. Kalra 


\title{
Recent advances in cardiac computed tomography dose reduction strategies: a review of scientific evidence and technical developments
}

\author{
Sandeep S. Hedgire, ${ }^{*}$ Vinit Baliyan, Brian B. Ghoshhajra, and Mannudeep K. Kalra \\ Massachusetts General Hospital, Department of Imaging, Division of Cardiovascular Imaging, Boston, Massachusetts, United States
}

\begin{abstract}
Cardiac imagers worldwide are bracing for increased utilization of cardiac computed tomography (CT) in clinical practice. This expanding opportunity brings along a responsibility to produce diagnostic quality images with optimized radiation dose. The following review aims to address the dose reduction strategies in cardiac CT in light of recent scientific evidence and technical developments. ๑ 2017 Society of Photo-Optical Instrumentation Engineers (SPIE) [DOI: 10.1117/1.JMI.4.3.031211]
\end{abstract}

Keywords: computed tomography; cardiac computed tomography; radiation dose; dose reduction; safety; dose optimization. Paper 17070SSVRR received Mar. 16, 2017; accepted for publication Jul. 28, 2017; published online Aug. $24,2017$.

\section{Introduction}

Rapid advances in scanner technology have established computed tomography (CT) as an indispensable tool for the assessment of cardiac anatomy, coronary circulation, and the great vessels. Expanding applications coupled with increasing use have raised concerns over potential stochastic effects of radiation dose associated with CT scanning, in particular, the risk of radiation-induced carcinogenesis. ${ }^{1,2}$ Indeed, the use of radiationbased medical imaging has exploded over the past few decades and CT has emerged as the dominant contributor to medical radiation dose, accounting for $\sim 46 \%$ of all the medical radiation dose to the United States population. ${ }^{3-7}$

Initial implementation of electrocardiographically (ECG) gated coronary CT angiography relied heavily on retrospectively gated acquisition mode, which was associated with relatively high radiation dose. ${ }^{8-11}$ Subsequent technologic evolution in both hardware and software CT technologies has helped lead a decreasing trend in mean radiation dose from cardiac CT. ${ }^{12}$ Several studies have demonstrated that the radiation dose from cardiac CT angiography (CCTA) can be reduced to lower than invasive catheter angiography with the use of techniques, such as prospective triggering, low tube potential technique, automatic exposure control (AEC), iterative reconstructive techniques, and so on. ${ }^{13-27}$ Conversely, despite the abundance of radiation dose-reduction strategies, several studies have reported a high variability in the radiation doses from cardiac CT. ${ }^{11,28-30}$ In an international, multicenter study, Hausleiter et al. ${ }^{11}$ reported that median doses from CCTA varied substantially among 21 participating institutions' different CT systems, and that radiation reduction strategies were not adequately utilized.

From the point of view of justification or appropriateness of CCTA, there is now a large body of evidence supporting its use in coronary artery disease including acute coronary syndromes. $^{31-36}$ There is a need for raising awareness for its appropriate use and deployment of dose-reduction strategies available

*Address all correspondence to: Sandeep S. Hedgire, E-mail: Hedgire .Sandeep@mgh.harvard.edu across different scan vendor platforms. We review various radiation dose-reduction strategies in the field of cardiac CT along with the scientific evidence in support of their value in the current clinical practice.

\section{Scanner and Patient-Related Factors in Cardiac Computed Tomography Angiography}

ECG-gated cardiac CT images are acquired/reconstructed at time points in the cardiac cycle when the heart is relatively motion free (end systole or middiastole). To detect and predict these critical time points, CT data are acquired simultaneously with recording of the ECG data. ECG tagging of CT data enables image reconstruction from data acquired during these critical phases of the cardiac cycle. Phase of a reconstructed dataset refers to the position of the image reconstruction window within the cardiac cycle. Radiation dose may be switched on only during these phases (prospectively ECG triggered) or continuously with selective data filtering from acquired data (retrospective ECG gated).

Prospective gating results in much lower radiation dose but cannot be applied with rapid heart rate with most of the scanners and can also result in stair-step artifacts and nonuniform contrast within the vessels. The stair-step artifact can also occur with ECG-gated cardiac CT due to gaps in data used for reconstruction. The prospectively triggered scanning does not provide full functional information; however, when cardiac functional information is obligatory, a prospectively ECG-triggered acquisition with a widened acquisition window (also referred to as "padding") can provide limited functional information.

The retrospective ECG gating provides multiple time points for image reconstruction, which allows functional assessment and may help mitigate the effects of motion artifacts, although at a cost of increased radiation dose. Prospective gating is applied in combination with sequential axial step and shoot scanning, whereas retrospective gating is applied in combination with a helical scanning mode. For helical scan mode, the pitch is defined as the ratio of table movement along the $z$-axis during 
single tube rotation and the beam width in the $z$-axis. ${ }^{8}$ Temporal resolution is a fundamental requirement of CCTA and requires fast-rotating gantries and multisegmented reconstructions (slice data acquired over more than one cardiac cycle). Faster gantry rotation and multisegmented reconstructions require a slower pitch in cardiac mode to avoid discontinuities in the anatomic coverage of the heart between images reconstructed from consecutive cardiac cycles. Multisegmented reconstruction is one of the most important factors responsible for high radiation dose. Due to radiation concerns and the limited clinical performance of multisegment reconstruction with variable heart rate, most scanners have these days switched to single-segment reconstruction. The helical pitch has to be still low in single-segment reconstruction, as the table should not move more than beam width during the time of one heart cycle to ensure gapless volume coverage. The pitch also has to be adapted to the patient's heart rate and needs to be lower at lower heart rates. This leads to a relatively higher radiation dose with retrospectively gated scans in patients with lower heart rates. At our institution, a prospective gating technique is typically used in patients with heart rates lower than 75 per minute on a 128-slice dual-source scanner.

\subsection{Other Scanner-Related Factors}

The radiation dose is primarily dependent on radiation output of the scanner, which is approximately proportional to the square of tube potential $(\mathrm{kVp})$ and the product of tube current and gantry rotation time (mAs). Several scan factors have a major impact on radiation dose by affecting pitch during CCTA. These include number of tubes (single versus dual source), gantry rotation time and $z$-axis coverage in single tube rotation (number of detector rows). Maintaining a low-pitch value is not necessary for dual-source $\mathrm{CT}$ and it could be increased to almost double at high heart rates allowing radiation dose savings. ${ }^{37}$ Recent scanners equipped with a large number of detector rows (256 to 320) may allow up to $16 \mathrm{~cm} z$-axis coverage, which is sufficient to cover the entire heart in single rotation eliminating the need of overlapping acquisitions. In combination with high gantry rotation speed/temporal resolution, these scanners allow single beat axial acquisition with a static table. ${ }^{38-41}$

The image reconstruction algorithms used for CT image reconstruction can further influence the choice of scan factors and thus the associated radiation dose. Most modern CT scanners are increasingly employing sophisticated iterative reconstruction (IR) techniques as compared with the previously used filtered back projection (FBP) algorithms, which are less computationally demanding. Advances in computation hardware and speed have enabled IR techniques into the mainstream to mitigate adverse effects of reduced radiation dose in FBP images while retaining diagnostic information. ${ }^{42}$

\subsection{Patient-Related Factors}

Radiation dose also depends on the body size as penetration of a larger body size demands a higher photon number and energy. ${ }^{43}$ Heart rate and rhythm often dictate the acquisition mode (prospective versus retrospective) and profoundly affect associated radiation dose with CCTA. Rapid heart rates (tachycardia) shorten the time window of relatively motion-free phases of the cardiac cycle (diastole), which can force the users to employ a retrospectively gated CCTA technique. Irregular rhythm with high heart rates may require application of the arrhythmia rejection technique (Fig. 1) with prospective triggering at end systole, which can result in increased radiation exposure (although still lower than a retrospectively gated scan). ${ }^{44-48}$

\section{General Radiation Dose-Reduction Strategies in Cardiac Computed Tomography}

\subsection{Clinical Indication-Driven Protocol}

Indications such as assessment of coronary calcification, pulmonary veins, and myocardium, can be scanned with lower spatial and temporal resolutions and with much lower radiation doses. When CCTA is being performed primarily for anomalous origin and course of coronary arteries, aggressive dose reduction can be applied without sacrificing diagnostic information. ${ }^{49}$

\subsection{Anatomic Scan Coverage (z-Axis)}

This is an important factor determining the radiation dose, which is directly proportional to $z$-axis coverage. ${ }^{43}$ Achieving optimal anatomic coverage for different indications requires careful planning. According to prior publications, each additional $1 \mathrm{~cm}$ of coverage at CCTA may increase the radiation dose by 40 to $100 \mathrm{mGy} \mathrm{cm}$, although the additional dose per $\mathrm{cm}$ might not be this high with modern state-of-the-art scanners. ${ }^{11,49}$ Appropriate scan coverage for CCTA may be achieved either on the basis of the initial calcium score scan or with the use of anatomic landmarks on the planning radiograph (tracheal bifurcation on the frontal view to the bottom of the cardiac silhouette on the lateral view). Leschka et al. ${ }^{50}$ reported that scan length adjustments on the basis of a calcium scoring scan may allow a $16 \%$ radiation dose reduction. Scan range for CCTA for evaluation of postcoronary bypass grafts may vary often extending from the origin of the left internal mammary artery (at the root of the neck) to the base of the heart. The coverage for pulmonary veins extends from $2 \mathrm{~cm}$ above the aortic arch to the dome of the diaphragm. ${ }^{49}$

\subsection{Automatic Exposure Control}

These techniques help adapt tube current according to the anatomic factors and specified image quality metric (angular, longitudinal, and combined types) or according to specified phases of ECG where reduced tube current is acceptable for image creation (ECG-based modulation). The angular AEC cannot be applied to ECG-gated scans but longitudinal AEC along the $z$-axis based on the planning radiographs can be applied in cardiac imaging and helps adapt tube current to changing body habitus. ${ }^{51}$

ECG-triggered prospective axial scanning is being increasingly applied instead of retrospective ECG-gated helical scanning for radiation dose reduction in CCTA examinations. ${ }^{18,52}$ For retrospective ECG-gated CCTA examinations, the evaluation of ventricular function does not require high quality and the tube current can be modulated according to the ECG signal, with the full tube current applied during the end-systolic or middiastolic phases and the tube current kept at lower levels during the rest of the cardiac cycle (retrospective gating with ECGbased modulation). ${ }^{53,54}$ Based on the CT vendor, tube current can be reduced to a user-specified minimum level or $4 \%$ to $20 \%$ of the tube current applied in the phases with higher image quality requirement. A variable amount of dose reduction is noted with application of this type of modulation based on the length 


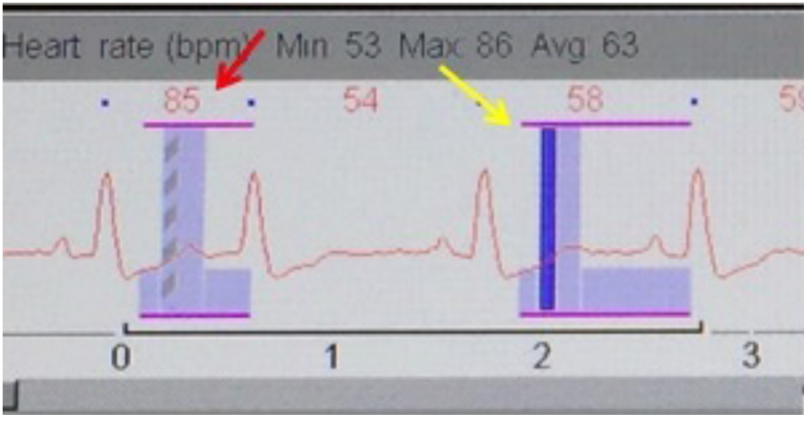

(a)

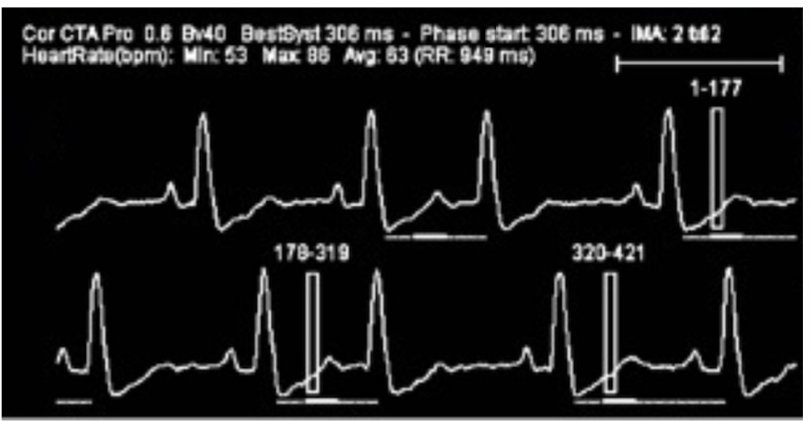

(b)

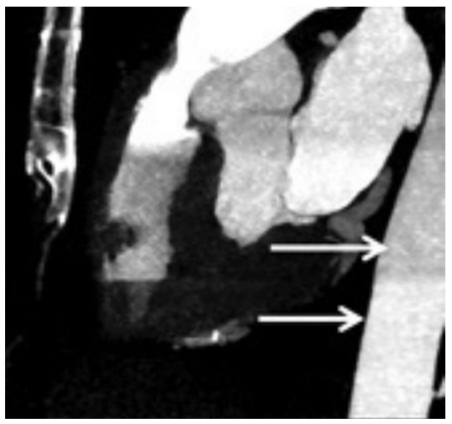

(c)

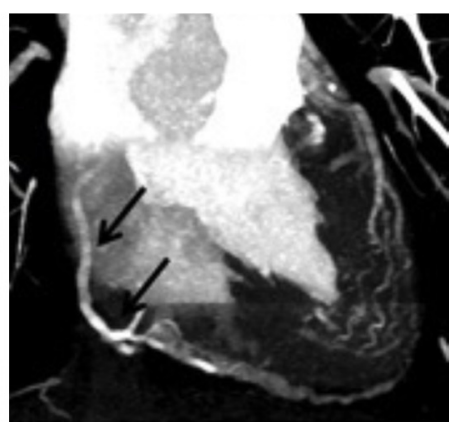

(d)

Fig. 1 Arrhythmia rejection: arrhythmia rejection software allows for repeated acquisition of the slab acquired during the arrhythmia/rapid heart rate variation-related beats. As shown in images (a, b), the first slab acquisition coincided with a short R-R interval (red arrow) and would have resulted in motion-related artifact on the final images. Arrhythmia rejection software detects it in real time and repeats the acquisition of that slab during next heart beat (yellow arrow). This technique helps in overall dose reduction by salvaging a scan that can be nondiagnostic and might have to be repeated with repeated dose of iodinated contrast material and additional radiation dose. The drawback of this strategy might be a nonuniform opacification of the coronaries and other vessels due to an extra step increasing the scan time (c, d; from a different patient).

of the phase over which higher image quality is desired, and heart rate. A rapid heart rate leaves a smaller duration of cardiac cycle over which reduced tube current can be applied and vice versa. Compared to the use of fixed tube current-based retrospectively gated CCTA, up to $40 \%$ reduction in radiation dose from CCTA can be achieved with ECG-based tube current modulation. ${ }^{55}$

\subsection{Tailoring Acquisition Modes to Heart Rate}

Heart rate and its rhythm can affect both the ability to obtain motion-free coronary artery evaluation and reduce radiation dose with appropriate acquisition mode (prospectively ECG triggered versus retrospectively ECG gated). A target heart rate of up to 75 beats per minute is desirable with most vendors to minimize cardiac-motion artifacts and also reduce radiation dose associated with CCTA. Such a regular low heart rate enables use of prospectively ECG-triggered axial or helical highpitched scan mode, which is associated with lower radiation dose (up to $90 \%$ lower) compared with retrospectively ECGgated helical scan mode. Heart rate control medications, such as beta blockers, are frequently administered to reduce heart rate to apply prospective triggering and maximize the number of evaluable coronary segments. Although the need for rate control strongly depends on the CT system used and its temporal resolution, the modern $\mathrm{CT}$ systems may provide ECG-triggered prospective scans for patients with heart rates up to $70 \mathrm{bpm}$ or even higher.

\subsection{Parameter Selection Based on Body Habitus}

AEC and automatic tube potential selection techniques for CCTA examinations should be used when available to apply appropriate tube potential and current based on the patient's body habitus. Most nonobese patients [Body mass index (BMI) of up to $30 \mathrm{~kg} / \mathrm{m}^{2}$ ] should be scanned $100 \mathrm{kV}$ instead of the traditional $120 \mathrm{kV}^{25,56,57}$ In morbidly obese patients (BMI $>35 \mathrm{~kg} / \mathrm{m}^{2}$ ), a combination of high potential $(\geq 120 \mathrm{kV})$, high tube current, higher strength of IR, and retrospective ECG gating may be needed to obtain acceptable image quality. ${ }^{49}$

\section{Recent Advances in Radiation Dose Reduction with Clinical Evidence}

Several notable developments have transformed CCTA and enhanced its role in cardiac imaging while enabling substantial radiation dose reduction. These include wide-area detector arrays, dual-source CT, automatic tube potential selection, adaptive prospective ECG triggering, and IR. CT scanners with wide-area detector arrays (e.g., Toshiba 320 to 640-slice, GE 256 -slice) enable $16 \mathrm{~cm}$ scan coverage of the entire heart in single gantry rotation and single heart beat, and eliminate banding or slab artifacts. The dual-source CT (64-, 128-, 192-slice, Siemens) has helped enhance the temporal resolution and enable high-pitch prospectively ECG-triggered helical mode of acquiring data in a single heart beat as well. In this section, we discuss some of these recent advances from the perspective of cardiac $\mathrm{CT}$ and radiation dose optimization. 


\subsection{Low Tube Potential}

When other scan factors are held constant, low tube potential results in radiation dose reduction ( $\operatorname{dose} \alpha V^{2}$ ) and decreased requirement of iodinated contrast, but with an increase in image noise. This noise can be tolerated for two reasons: first, due to close proximity with the $k$ edge of iodinated contrast, low tube potential results in high contrast and secondly effective reconstruction algorithms, such as IR techniques. ${ }^{58,59}$ The application of lower tube potential requires careful adjustment of the tube output (mAs) to maintain the desired contrast to noise ratio, leading to high $\mathrm{mAs}$ demands in particular for bigger patients. Hence, the use of low $\mathrm{kV}$ scanning has to be limited to patients with smaller body size. To decrease the impact of higher image noise on image quality a large window width can be used on the monitor display for image interpretation. Combined low-voltage tube and IR techniques can also help in maintaining good signalto-noise ratio comparable to using standard voltage tube and FBP techniques (Fig. 2). ${ }^{60}$

Low tube potential ( 80 or $100 \mathrm{kV}$ ) imaging for cardiac imaging was initially described for nonobese patients (BMI < $20 \mathrm{~kg} / \mathrm{m}^{2}, 80 \mathrm{kV}$; BMI 20 to $30 \mathrm{~kg} / \mathrm{m}^{2}, 100 \mathrm{kV}$ ) (Table 1). ${ }^{61}$ Experience from several clinical trials, such as PROTECTION I, PROTECTION II, and REALISE, demonstrated 53\%, 31\%, and $35 \%$ reductions in radiation dose, respectively, without substantial impairment of diagnostic image quality when the tube potential was lowered from 120 to $100 \mathrm{kV}$. $^{25,56,57}$ These studies were done with the use of 64-slice dual-source scanners and IR techniques. Based on these observations, current guidelines recommend the use of low tube potential ( 80 to $100 \mathrm{kV}$ ) for CCTA in nonobese patients.

Two recent studies have reported further dose reductions and diagnostic image quality with further lowering of tube potential
$70 \mathrm{kV}$ in nonobese patients ${ }^{62,63}$ on the third-generation dualsource scanners (Siemens Somatom Force), which enables much higher tube current (up to $1300 \mathrm{~mA}$ for each $\mathrm{x}$-ray tube) and IR technique (admire) ${ }^{63}$ On the same scanner, Mangold et al. ${ }^{64}$ reported diagnostic image quality for CCTA in overweight and obese patients at $120 \mathrm{kV}$, which were previously scanned with $>120 \mathrm{kV}$. Evaluating 102 overweight and obese patients with BMI ranging from 25 to $40 \mathrm{~kg} / \mathrm{m}^{2}$, authors demonstrated diagnostic quality CCTA and triple rule out evaluation with $120 \mathrm{kV} .{ }^{64}$ Zhang et al. ${ }^{65}$ have reported the use of $70 \mathrm{kV}$ with a 64-slice dual-source scanner, but these results must be interpreted cautiously as more than three quarters of the included patients had $\mathrm{BMI} \leq 25 \mathrm{~kg} / \mathrm{m}^{2}$ and there was a reduction in diagnostic accuracy on a per-patient, per-vessel, and persegment basis. Lower tube potentials are also associated with increased attenuation of iodine on contrast CT. Therefore, low tube potential protocols might potentially reduce the contrast dose and are also more suitable for cardiovascular applications, such as myocardial CT, perfusion to better detect the regional differences in myocardial contrast attenuation.

\subsection{High-Pitch Helical Acquisitions}

Single-source multidetector-row helical CT scanners are restricted to a pitch of up to $1.5: 1$ (range 0.2 to $1.5: 1$ ). The two simultaneous overlapping helices on dual-source CT with ECGtriggered prospectively gating enable image reconstruction at a high pitch of over 3:1 at significantly lower than the radiation dose. ${ }^{66,67}$ High-pitch helical (HPH) acquisition mode with fast table speed and prospective ECG triggering enables coverage of the entire heart within one cardiac cycle. Several studies on HPH mode for CCTA in pediatric and adult populations ${ }^{16,63,68-70}$ have reported good diagnostic quality and consistently low mean
120

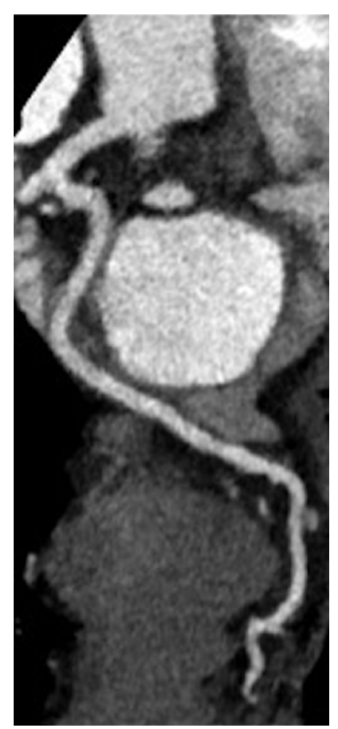

(a)
100

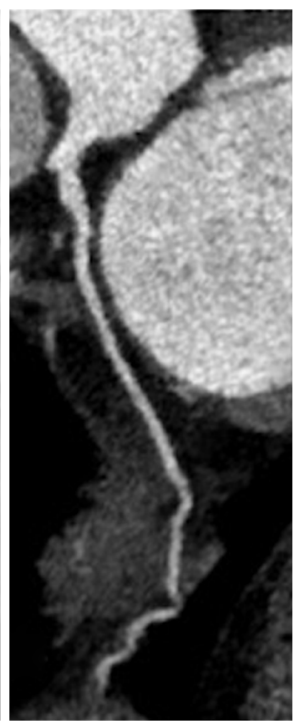

(b)
80

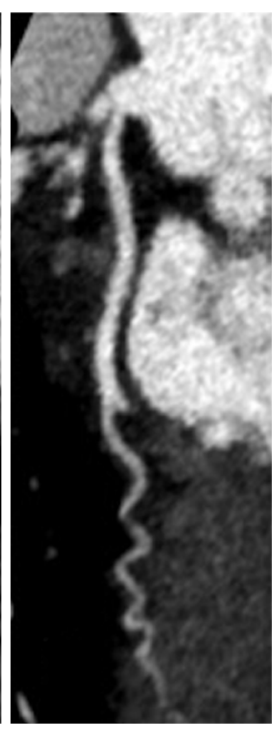

(c)
70

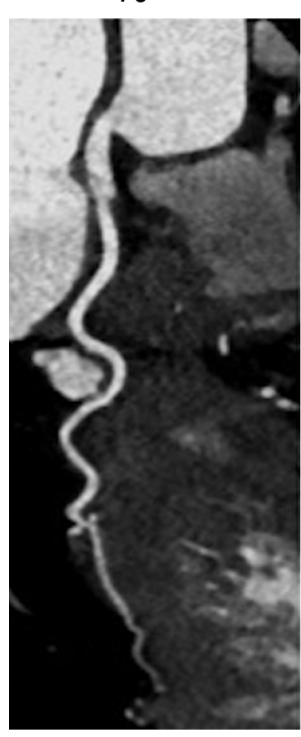

(d)

Fig. 2 Low kVp technique: Images (a) to (d) have been acquired with 120, 100, 80, and $70 \mathrm{kVp}$, respectively, and demonstrate diagnostic image quality at all $k V p$ levels. Images a to $c$ were acquired on a 128 $(\times 2)$ slice dual-source scanner and with image reconstruction using IR-level 3 . The image "d" has been acquired with $70 \mathrm{kVp}$ on a $192(\times 2)$ slice third-generation dual-source scanner on a patient with BMI comparable to $100 \mathrm{kVp}$ image (IR-level 3 reconstruction). The third-generation dual-source scanner is capable of generating very high $\mathrm{mA}(2 \times 1300 \mathrm{~mA})$ allowing for acceptable image quality and low radiation dose at $\mathrm{kVp}$ as low as 70 . The DLP for 100 (current standard for CCTA) and $70 \mathrm{kVp}$ acquisitions were 404 and $192 \mathrm{mGy} \mathrm{cm}$, respectively. 
Hedgire et al.: Recent advances in cardiac computed tomography...

Table 1 Cardiac CT at low tube potential imaging: recent evidence (key: R, retrospective; P, prospective; DSCT, dual-source CT; MC, multicenter).

\begin{tabular}{|c|c|c|c|c|c|}
\hline Author and year & Study & \# patients & Scanner platform & $\mathrm{kV}$ & Radiation dose \\
\hline $\begin{array}{l}\text { Bischoff et al. } \\
\text { (PROTECTION I) } \\
(2009)^{56}\end{array}$ & P-MC & $\begin{array}{c}321 \\
\text { (nonobese) }\end{array}$ & $\begin{array}{l}16 \text { or } 64 \text { slice } \mathrm{CT} \\
\text { (multiple platforms) }\end{array}$ & 100 and $120 \mathrm{kV}$ & $\begin{array}{l}\text { CTDIvol @ } 120 \mathrm{kV}=52 \mathrm{mGy} \\
\text { CTDIvol @ } 100 \mathrm{kV}=24 \mathrm{mGy}\end{array}$ \\
\hline $\begin{array}{l}\text { Hausleiter et al. } \\
\text { (PROTECTION II) } \\
(2010)^{25}\end{array}$ & P-MC & $\begin{array}{c}400 \\
\text { (nonobese) }\end{array}$ & $\begin{array}{l}8 \text { sites 64-slice CT } \\
\text { (GE, Siemens, Toshiba) }\end{array}$ & 100 and $120 \mathrm{kV}$ & $\begin{array}{l}\text { CTDIvol @ } 120 \mathrm{kV}=63 \pm 26 \mathrm{mGy} \\
\text { CTDIvol @ } 100 \mathrm{kV}=43 \pm 20 \mathrm{mGy}\end{array}$ \\
\hline Meyer et al. $(2014)^{62}$ & $P$ & 45 (nonobese) & $\begin{array}{l}\text { 192-slice DSCT and } \\
\text { 128-slice DSCT }\end{array}$ & $\begin{array}{c}\text { 192-CT: } 70 \mathrm{kV} \\
\text { 128-CT: } 80 \text { and } 100 \mathrm{kV}\end{array}$ & $\begin{array}{l}\text { CTDIvol @ } 100 \mathrm{kV}=1.6 \pm 0.4 \mathrm{mGy} \\
\text { CTDIvol @ } 80 \mathrm{kV}=1.1 \pm 0.3 \mathrm{mGy} \\
\text { CTDIvol @ } 70 \mathrm{kV}=1.2 \pm 0.1 \mathrm{mGy}\end{array}$ \\
\hline $\begin{array}{l}\text { Yin et al. (REALISE) } \\
(2015)^{57}\end{array}$ & P-MC & 231 & 128-slice DSCT & $\begin{array}{l}\text { FBP: } 120-k V \text { and } \\
\text { IRT: } 100 \mathrm{kV}\end{array}$ & $\begin{array}{l}\text { SSDE @ } 120 \mathrm{kV}=27 \pm 8 \mathrm{mGy} \\
\text { SSDE @ } 100 \mathrm{kV}=18 \pm 7 \mathrm{mGy}\end{array}$ \\
\hline Andreini et al. $(2016)^{61}$ & $P$ & $105(<80 \mathrm{Kg})$ & $\begin{array}{l}64 \text { slice (discovery } \\
\text { CT } 750 \text { HD) }\end{array}$ & $80 \mathrm{kV}$ & Mean DLP $=79 \pm 33$ mGy cm \\
\hline Mangold et al. $(2016)^{64}$ & $\mathrm{R}$ & $\begin{array}{l}110 \text { (obese } \\
\text { and nonobese) }\end{array}$ & 128-slice DSCT & $\begin{array}{c}70,80,90,100 \\
110,120 \mathrm{kV}\end{array}$ & $\begin{array}{l}\text { CTDlvol < } 120 \mathrm{kV}: 13 \pm 12 \mathrm{mGy} \\
120 \mathrm{kV}: 37 \pm 13 \mathrm{mGy}\end{array}$ \\
\hline
\end{tabular}

radiation dose (0.8 to $1.3 \mathrm{mSv}$ ) (Table 2$)$. The $\mathrm{HPH}$ is particularly advantageous in children or young adults for assessment of coronary artery anomalies and anatomic assessment in patients with congenital heart disease (when information regarding cardiac function is not needed). In particular, HPH can help avoid need for sedation or anesthesia in young children due to its rapidity.

Important limitations of HPH mode include inconsistent diagnostic quality in patients with increased heart rates, ${ }^{16,68}$ motion artifacts and nonapplicability of retrospective reconstruction, and functional assessment of the heart. ${ }^{71}$ To mitigate the effects of motion on evaluation of coronary artery evaluation, a prior study has recommended use of subsequent acquisitions in two different cardiac cycles. ${ }^{71}$ This technique is typically avoided in morbidly obese patients with suspected or known coronary atherosclerotic disease due to higher likelihood of artifacts and excessive image noise.

\subsection{Iterative Reconstructions}

The iterative reconstructions (IR) techniques have had a major impact on reducing radiation dose and have found applications in all domains of CT imaging. Today, major CT vendors offer multiple IR techniques on different generations of their scanners. Indeed, IR techniques are replacing conventional FBP techniques since they are less prone to artifacts and increased image noise at lower radiation doses as compared with FBP, which may have compromised diagnostic quality at low radiation doses. ${ }^{42,72}$

IR techniques remove image noise through a process of modeling of the imaging acquisition processes including photon statistics fluctuations, the optics system, and other aspects of $\mathrm{x}$-ray interactions that are ignored by the conventional FBP techniques. IR techniques use and include a simple algebraic reconstruction technique, statistical IR, and more recent model-based IR (MBIR). Current IR algorithms typically combine statistical modeling with MBIR. ${ }^{73}$ Multiple studies have evaluated the impact of IR techniques on image quality and radiation dose for different clinical indications in both adults and pediatric patients including CCTA (Table 3). ${ }^{13-15,74,75} \mathrm{~A}$ recent metaanalysis reported that the IR techniques can lower radiation dose by up to $48 \%$ in comparison with the traditional FBP method for CCTA. ${ }^{14}$ Availability and application of IR in CCTA have helped in applying lower tube potential and tube current for reducing radiation dose while improving image contrast.

Table 2 HPH acquisition mode in cardiac CT, recent evidence (key: R, retrospective; P, prospective; DSCT, dual-source CT; SSCT, single source CT; proECG, prospectively ECG-triggered axial mode; retroECG, retrospectively ECG-gated mode).

\begin{tabular}{|c|c|c|c|c|c|}
\hline Author & Study and year & \# patients & Scanner platform & Pitch & Dose and comments \\
\hline Achenbach et al. ${ }^{68}$ & $\mathrm{P}$ and 2010 & 69 & 128-slice DSCT & 3.2 to $3.4: 1$ & $\begin{array}{l}\text { DLP } 3.2 \text { pitch: } 67 \pm 3 \mathrm{mGy} \mathrm{cm} \\
3.4 \text { pitch: } 57 \pm 2 \mathrm{mGy} \mathrm{cm}\end{array}$ \\
\hline Ghoshhajra et al. ${ }^{69}$ & $\mathrm{R}$ and 2014 & 95 & $\begin{array}{l}\text { 64-slice SSCT 64-slice } \\
\text { DSCT 128-slice DSCT }\end{array}$ & 3.2 to $3.4: 1$ & $\begin{array}{l}\text { DLP (median doses) } \mathrm{HPH}=49 \mathrm{mGy} \mathrm{cm} \\
\text { proECG }=136 \mathrm{mGy} \mathrm{cm} \text { retroECG }= \\
212 \mathrm{mGy} \mathrm{cm}\end{array}$ \\
\hline Selcuk et al. ${ }^{70}$ & $\mathrm{R}$ and 2016 & 450 & 128-slice DSCT & $3.2: 1$ & DLP $74 \pm 7$ mGy cm \\
\hline Linsen et al. ${ }^{63}$ & $\mathrm{R}$ and 2016 & 100 & $\begin{array}{l}\text { 128-slice DSCT: } 100 / 120 \mathrm{kV} \\
\text { 192-slice DSCT: } 70 / 120 \mathrm{kV}\end{array}$ & 3.2 to $3.4: 1$ & $\begin{array}{l}\text { DLP 128-slice DSCT: } 84 \pm 33 \mathrm{mGy} \mathrm{cm} \\
\text { 192-slice DSCT: } 41 \pm 21 \mathrm{mGy} \mathrm{cm}\end{array}$ \\
\hline
\end{tabular}


Table 3 Iterative reconstruction in cardiac CT: recent evidence (key: R, retrospective; $\mathrm{P}$, prospective; M, meta-analysis; DSCT, dual-source CT).

\begin{tabular}{|c|c|c|c|c|c|}
\hline Author and year & Study and year & \# patients & Scanner platform & Iterative algorithm & Radiation dose \\
\hline Yin et al. ${ }^{74}$ & $\mathrm{P}$ and 2013 & 60 & $\begin{array}{l}\text { 128-slice DSCT } \\
\text { (definition flash) }\end{array}$ & $\begin{array}{l}\text { Full mAs (FBP) versus } \\
50 \% \text { less } \mathrm{mAs}(\mathrm{IR})\end{array}$ & $\begin{array}{l}\text { CTDIvol IR: } 1.2 \pm 0.5 \text { mGy FBP: } \\
2.4 \pm 1 \text { mGy }\end{array}$ \\
\hline Benz et al. ${ }^{13}$ & $P(2016)$ & 65 & $\begin{array}{l}\text { 256-slice MDCT } \\
\text { (GE revolution) }\end{array}$ & ASiR-V (6 levels) & Median DLP: $35 \mathrm{mGy} \mathrm{cm}$ \\
\hline $\begin{array}{l}\text { Den Harder } \\
\text { et al. }{ }^{14}\end{array}$ & M (2016) & $\begin{array}{l}1042 \text { from } \\
10 \text { studies }\end{array}$ & $\begin{array}{l}\text { 64-320 slice MDCT (GE, } \\
\text { Philips, Siemens, Toshiba) }\end{array}$ & $\begin{array}{l}\text { ASIR, MBIR iDose } \\
\text { IRIS, Safire AIDR 3D }\end{array}$ & $\begin{array}{l}\text { CTDIvol, DLP, or SSDE not provided } \\
\text { but } 48 \% \text { lower dose with IR compared } \\
\text { to FBP }\end{array}$ \\
\hline lyama et al. ${ }^{15}$ & $P(2016)$ & 60 & $\begin{array}{l}\text { 256-slice CT (Philips } \\
\text { Brilliance iCT) }\end{array}$ & $\begin{array}{l}80 \mathrm{kV} \text { IMR versus } \\
120 \mathrm{kV} \text { FBP }\end{array}$ & $\begin{array}{l}80 \mathrm{kVp} \text { IMR (CTDIvol }=8 \pm 0.1 \mathrm{mGy}) \\
120 \mathrm{kVp} \text { FBP (CTDIvol }=29 \pm 0.2 \mathrm{mGy})\end{array}$ \\
\hline
\end{tabular}

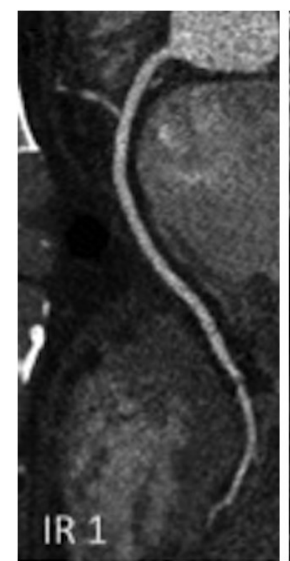

(a)

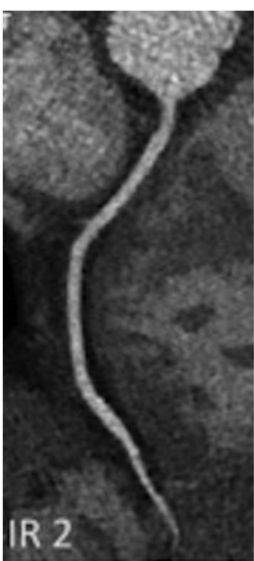

(b)

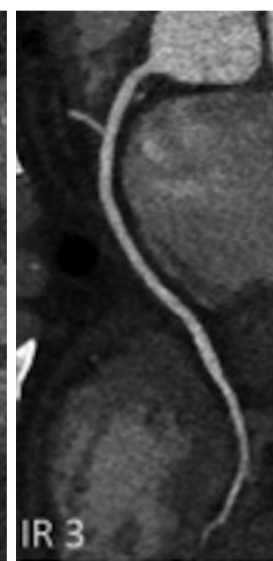

(c)

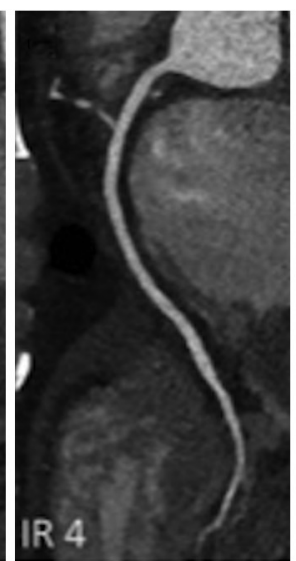

(d)

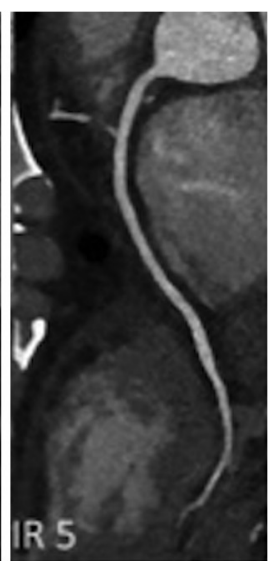

(e)

Fig. 3 Hybrid IR: application of increasing strengths of iterative reconstruction results in progressive noise reduction. The combination of IR compensates for the high intrinsic image noise from low $\mathrm{kV}$ technique. The images (a) to (e) are demonstrating a progressive noise reduction with increasing strength of IR reconstruction. We typically use images of IR-level 3 for our routine clinical work in combination with applicative of low $\mathrm{kV}$ acquisition technique.

There are several types of IR techniques available for commercial use but the most commonly used one involves blending of IR and FBP techniques (hybrid). Hybrid IR techniques involve prefixed blending of FBP and IR data and contrast to noise ratio has been shown to significantly improve with increasing strength or level of IR contribution (Fig. 3). ${ }^{76}$ A common concern or limitation at higher strength or level of IR algorithms pertains to the fact that radiologists might not feel comfortable with rather "glossy" images with increasing IR levels, which may be mitigated with increasing familiarity with the IR. ${ }^{77}$ The "glossy" appearance is more pronounced with pure model-based IR techniques, which also take substantially longer reconstruction time compared with hybrid IR techniques.

\subsection{Single Heart Beat Scan with Wide Detector Array}

Other technical evolution in recent times has been the development of wide detector array scanners (up to $16 \mathrm{~cm}$ detector width along the patient length) with full cardiac coverage in single gantry rotation and single heart beat. These scanners eliminate banding artifacts (previously known as slab or misalignment artifacts) and provide uniform contrast enhancement in the coronary arteries. They also allow measurement of contrast attenuation gradients across atherosclerotic lesions (information somewhat similar to functional flow reserve) and are better suited for myocardial perfusion assessment. ${ }^{12}$ Common concerns pertaining to these scanners include the need for full 360-deg rotation, relatively higher radiation dose and cone beam geometry-related artifacts.

Various studies have reported radiation doses associated with the use of wide detector array MDCT scanners for CCTA ranging from 4.4 to $15 \mathrm{mSv}$., $38,39,41,78,79$ In a single study with second-generation wide-volume 320-detector row MDCT, Chen et al. $^{40}$ reported a substantially low radiation dose for CCTA examinations $(0.9 \mathrm{mSv}$; interquartile range 0.6 to $1.7 \mathrm{mSv})$.

\section{Pediatric Cardiac Computed Tomography}

Due to higher radiation risk and the need for serial followup imaging, pediatric cardiac CT warrants special attention. Echocardiography has a limited role in the characterization of complex congenital heart disease and coronary evaluation beyond proximal segments. MRI is an excellent modality for functional assessment; however, its use can also be limited by the presence of noncompatible devices, need for anesthesia, and long image acquisition time. ${ }^{80}$ Armed with technologic 
Hedgire et al.: Recent advances in cardiac computed tomography...

Table 4 Pediatric cardiac CT: prospective studies on radiation dose reduction while maintaining diagnostic information. (Key: R, retrospective; P, prospective; M, meta-analysis; DSCT, dual-source CT; proECG, prospectively ECG-triggered axial mode; retroECG, retrospectively ECG-gated mode).

\begin{tabular}{|c|c|c|c|c|}
\hline Author and year & \# patients & Scanner platform & Techniques employed & Radiation dose \\
\hline Hou et al. $(2017)^{20}$ & 110 & $\begin{array}{l}\text { 64-slice MDCT (GE } \\
\text { discovery } 750 \mathrm{HD})\end{array}$ & $\begin{array}{l}80 \mathrm{kVp} 70 \% \mathrm{IR} 120 \mathrm{~mA} \\
(n=45) 160 \mathrm{~mA}(n=65)\end{array}$ & $\begin{array}{l}\text { DLP } 120 \mathrm{~mA}: 15 \pm 1.9 \mathrm{mGycm} \\
160 \mathrm{~mA}: 20 \pm 2.1 \mathrm{mGy} \mathrm{cm}\end{array}$ \\
\hline Koplay et al. $(2016)^{81}$ & 105 & $\begin{array}{l}\text { 128-slice DSCT (definition } \\
\text { flash, Siemens) }\end{array}$ & $\begin{array}{l}3.4: 1 \text { pitch } 70 \mathrm{kVp} \text { AEC } \\
\text { (80 to } 140 \mathrm{mAs}) \text { Safire (IR) }\end{array}$ & $\begin{array}{l}\text { CTDIvol } 0.3 \pm 0.1 \mathrm{mGy} \text { DLP: } \\
16 \pm 10 \mathrm{mGy} \mathrm{cm}\end{array}$ \\
\hline Gao et al. $(2016)^{82}$ & 102 & $\begin{array}{l}\text { 64-slice MDCT (discovery } \\
750 \mathrm{HD}, \mathrm{GE})\end{array}$ & $\begin{array}{l}80 \mathrm{kVp} \text { AEC } 35-42 \mathrm{mAs} \\
80 \% \text { ASIR }\end{array}$ & $\begin{array}{l}\text { DLP proECG: } 14 \pm 4 \mathrm{mGy} \mathrm{cm} \\
\text { retroECG: } 51 \pm 9 \mathrm{mGy} \mathrm{cm}\end{array}$ \\
\hline Yang et al. $(2017)^{75}$ & 160 & $\begin{array}{l}\text { 256-slice MDCT (Philips } \\
\text { Brilliance iCT) }\end{array}$ & $\begin{array}{l}\text { Half mAs iDose }{ }^{4}(\mathrm{IR}) \\
\text { compared to full dose FBP }\end{array}$ & $\begin{array}{l}\text { CTDIvol IR: } 3.6 \pm 1 \mathrm{mGy} \text { FBP: } \\
1.7 \pm 0.5 \mathrm{mGy}\end{array}$ \\
\hline
\end{tabular}

Table 5 Additional literature on reducing radiation dose for CCTA (key: R, retrospective; P, prospective; DSCT, dual-source CT; NA, not applicable; proECG, prospectively ECG-triggered axial mode; retroECG, retrospectively ECG-gated mode; HPH, helical high pitch).

\begin{tabular}{|c|c|c|c|c|c|}
\hline Author & $\begin{array}{l}\text { Study design } \\
\text { and year }\end{array}$ & \# Patients & Scanner platform & Novel technique employed & Radiation dose \\
\hline Lee et al. ${ }^{48}$ & R (2012) & 90 & $\begin{array}{l}\text { 128-slice DSCT } \\
\text { (definition flash) }\end{array}$ & Adaptive proECG & $\begin{array}{l}\text { DLP (median) proECG: } 307 \text { mGy cm } \\
\text { retroECG: } 650 \text { mGy cm }\end{array}$ \\
\hline Andreini et al. ${ }^{84}$ & $P(2015)$ & 120 & $\begin{array}{l}\text { 64-slice MDCT } \\
\text { (discovery } 750 \mathrm{HD} \text { ) }\end{array}$ & $\begin{array}{l}\text { Motion-correction (MC) } \\
\text { algorithm }\end{array}$ & $\begin{array}{l}\text { DLP } 80 \text { ms padding: } 170 \pm 66 \mathrm{mGy} \mathrm{cm} \\
200 \mathrm{~ms} \text { padding: } 310 \pm 102 \mathrm{mGy} \mathrm{cm}\end{array}$ \\
\hline Vadvala et al. ${ }^{85}$ & R (2014) & 726 & $\begin{array}{l}\text { 128-slice DSCT } \\
\text { (definition flash) }\end{array}$ & $\begin{array}{l}\text { Breast displacement in } \\
\text { female patients }\end{array}$ & $\begin{array}{l}\text { CTDI vol (median) females: } 8.6 \text { mGy } \\
\text { males: } 12.7 \mathrm{mGy}\end{array}$ \\
\hline Bischoff et al. ${ }^{88}$ & $P(2016)$ & 36 & $\begin{array}{l}\text { 128-slice DSCT } \\
\text { (definition flash) }\end{array}$ & $\begin{array}{l}\text { Combining CCTA and CT } \\
\text { perfusion in dense coronary } \\
\text { calcification or stent }\end{array}$ & $\begin{array}{l}\text { DLP stress CT perfusion (HPH): } \\
65 \pm 35 \text { mGy cm rest CT perfusion } \\
\text { (proECG): } 361 \pm 200 \text { mGy cm }\end{array}$ \\
\hline
\end{tabular}

advances, cardiac CT has emerged as a useful alternative in pediatric heart diseases.

As discussed above, most radiation dose-reduction techniques employed in adults are also applicable in children (Table 4). Most children can be and should be scanned at lower tube potential $(70$ to $80 \mathrm{kV})$ and reduced tube current. ${ }^{20,81}$ When information pertaining to cardiac function is not required or can be obtained with other imaging modalities, prospectively ECGtriggered axial or helical mode (such as high pitch on dualsource CT) should be used along with lower tube potential and IR techniques in order to reduce radiation dose. Most CCTA can be routinely obtained at sub-mSv radiation doses in children using prospective ECG-triggered high-pitch DSCT and iterative reconstruction to achieve consistent diagnostic scans..$^{20,69,75,81,82}$ When cardiac functional information is obligatory, either prospectively ECG-triggered acquisition with a widened acquisition window (also referred to as "padding") or retrospectively ECG-gated acquisition with an aggressive ECG-based tube current modulation should be used along with lower tube potential and optimal scan coverage. ${ }^{69}$

\section{Miscellaneous Dose-Reduction Strategies}

In this section, we discuss several other measures and techniques that can aid in optimizing radiation dose for CT (Table 5). ${ }^{63,70,83}$ Scanners with faster rotation times (better temporal resolution) or an increased number of x-ray sources permit higher heart rate cutoffs for prospectively ECG-triggered acquisition mode, which is associated with substantially lower radiation dose as compared with retrospectively ECG-gated acquisition mode. ${ }^{63,70,83}$ Addition of "adaptive" or arrhythmia rejection feature in prospectively ECG-triggered acquisition mode enables the scanners to reject scanning in beats with irregular rhythm so that obtain diagnostic quality exams at reduced radiation dose even in patients with variable heart rates, such as atrial fibrillation. Without the arrhythmia rejection algorithms for prospectively ECG-triggered mode, patients with variable heart rates would require much higher radiation doses from retrospectively ECG-gated acquisition mode with conservative ECG-based tube current modulation. In fact, use of adaptive prospectively ECG-triggered acquisition mode in these patients can decrease radiation dose by up to $50 \%$ compared with retrospectively ECG-gated helical DSCT. ${ }^{48}$ Motioncorrection algorithms can improve CCTA image quality and diagnostic capability in patients with high HR and HR variability at low radiation dose prospective scans. ${ }^{84}$

In female patients, radiation dose for cardiac CT can be substantially reduced by displacing the mobile portion of breasts using straps attached to the scanner table - a routinely performed exercise at our institution prior to acquisition of planning radiographs. ${ }^{85}$ In addition to dose reduction, this can help decrease image noise and/or artifacts in female patients with 
large breasts. Application of a bismuth-based breast displacement shield can effectively reduce breast exposure at the expense of increased noise and artifacts. Moreover, comparable breast radiation reductions can be achieved without shielding by globally reducing tube current with similar increase in image noise. At our institution, we neither use nor recommend the use of a bismuth shield for patients undergoing cardiac CT due to artifacts from edges of the bismuth shield. Bismuth shields can also affect the measured CT numbers, which is especially important in calcium scoring scans. To avoid these errors, a thick padding material needs to be placed between the patient's skin and the shield, a time-consuming and cumbersome method.

The more advanced methods of reducing exposure, including dose modulation and iterative reconstruction techniques, are superior to breast shielding and should be preferably used if available. ${ }^{86,87}$

Well-known limitations of CCTA pertain to evaluation of luminal stenosis in patients with dense coronary calcifications and stents. Recent studies have reported that combining CCTA and CT perfusion can provide better diagnostic accuracy for detecting hemodynamically significant coronary artery stenosis compared with CCTA alone in patients with dense coronary calcifications or stents. ${ }^{88,89}$ Dose reduction for such combined studies was achieved with a combination of low tube potential and prospectively ECG-triggered axial (for rest perfusion) and highpitch (for stress perfusion) acquisition mode. Dual energy CT has also been evaluated for myocardial perfusion. ${ }^{90}$ Comparable radiation doses between single- and dual-energy CT have been reported although further research is necessary to compare doses with low tube potential single energy $\mathrm{CT} .{ }^{90}$ Ability to provide additional information from generation of perfusion data might help in decreasing the need of additional radiation-based investigations and reduction in overall radiation dose.

Finally, the dose tracking initiative of the American College of Radiology Dose Index Registry warrants special attention. The registry provides an opportunity for CT centers and institutions to compare their radiation doses with other practices and then tailor cardiac CT protocols according to median or average radiation doses across the United States. ${ }^{91}$

\section{Conclusion}

Limiting radiation dose from $\mathrm{CT}$ is an important goal for the imaging community. Aforementioned techniques and strategies have helped in substantial reduction of radiation dose for cardiac CT examinations and make it an attractive and meaningful option for imaging suitable patients with suspected or known cardiac and coronary abnormalities.

\section{Disclosures}

No conflicts of interest, financial or otherwise, are declared by the authors SH, VB, and MK. Dr. BG serves as Educational consulting for Medtronic and Siemens.

\section{References}

1. D. J. Brenner and E. J. Hall, "Computed tomography-an increasing source of radiation exposure," N. Engl. J. Med. 357(22), 2277-2284 (2007).

2. D. J. Brenner and H. Hricak, "Radiation exposure from medical imaging: time to regulate?" JAMA 304(2), 208-209 (2010).

3. F. A. Mettler et al., "Effective doses in radiology and diagnostic nuclear medicine: a catalog," Radiology 248(1), 254-263 (2008).
4. H. Hricak et al., "Managing radiation use in medical imaging: a multifaceted challenge," Radiology 258(3), 889-905 (2011).

5. F. A. Mettler et al., "Radiologic and nuclear medicine studies in the United States and worldwide: frequency, radiation dose, and comparison with other radiation sources-1950-2007," Radiology 253(2), 520-531 (2009)

6. E. C. Lin, "Radiation risk from medical imaging," Mayo Clin. Proc. 85(12), 1142-1146 (2010).

7. J. A. Brink and R. L. Morin, "Size-specific dose estimation for CT: how should it be used and what does it mean?" Radiology 265(3), 666-668 (2012).

8. B. Desjardins and E. A. Kazerooni, "ECG-gated cardiac CT," Am. J. Roentgenol. 182(4), 993-1010 (2004).

9. Z. Sun, G. H. Choo, and K. H. Ng, "Coronary CT angiography: current status and continuing challenges," Br. J. Radiol. 85(1013), 495-510 (2012).

10. A. J. Einstein, M. J. Henzlova, and S. Rajagopalan, "Estimating risk of cancer associated with radiation exposure from 64-slice computed tomography coronary angiography," JAMA 298(3), 317-323 (2007).

11. J. Hausleiter et al., "Estimated radiation dose associated with cardiac CT angiography," JAMA 301(5), 500-507 (2009).

12. A. Aghayev et al., "Recent developments in the use of computed tomography scanners in coronary artery imaging," Expert Rev. Med. Devices 13(6), 545-553 (2016).

13. D. C. Benz et al., "Adaptive statistical iterative reconstruction-V: impact on image quality in ultralow-dose coronary computed tomography angiography," J. Comput. Assisted Tomogr. 40(6), 958-963 (2016).

14. A. M. Den Harder et al., "Dose reduction with iterative reconstruction for coronary CT angiography: a systematic review and meta-analysis," Br. J. Radiol. 89(1058), 20150068 (2016).

15. Y. Iyama et al., "Submillisievert radiation dose coronary CT angiography," Acad. Radiol. 23(11), 1393-1401 (2016).

16. M. Koplay et al., "Radiation dose and diagnostic accuracy of high-pitch dual-source coronary angiography in the evaluation of coronary artery stenoses," Diagn. Interventional Imaging 97(4), 461-469 (2016).

17. Y. Nishiyama et al., "Effect of the forward-projected model-based iterative reconstruction solution algorithm on image quality and radiation dose in pediatric cardiac computed tomography," Pediatr. Radiol. 46(12), 1663-1670 (2016).

18. B. Bischoff et al., "Comparison of sequential and helical scanning for radiation dose and image quality: results of the prospective multicenter study on radiation dose estimates of cardiac CT angiography (PROTECTION) I study," Am. J. Roentgenol. 194(6), 1495-1499 (2010).

19. S. Deseive et al., "Image quality and radiation dose of a prospectively electrocardiography-triggered high-pitch data acquisition strategy for coronary CT angiography: the multicenter, randomized PROTECTION IV study," J. Cardiovasc. Comput. Tomogr. 9(4), 278-285 (2015).

20. Q.-R. Hou et al., "A prospective evaluation of contrast and radiation dose and image quality in cardiac CT in children with complex congenital heart disease using low-concentration iodinated contrast agent and low tube voltage and current," Br. J. Radiol. 90(1070), 20160669 (2017).

21. A. D. Choi et al., "Prospective evaluation of the influence of iterative reconstruction on the reproducibility of coronary calcium quantification in reduced radiation dose 320 detector row CT,' J. Cardiovasc. Comput. Tomogr. 10(5), 359-363 (2016).

22. G. Sun et al., "Application of low tube voltage coronary CT angiography with low-dose iodine contrast agent in patients with a BMI of $26-30 \mathrm{~kg}$ / $\mathrm{m}^{2}$," Clin. Radiol. 70(2), 138-145 (2015).

23. J. Stehli et al., "Accuracy of coronary CT angiography using a submillisievert fraction of radiation exposure: comparison with invasive coronary angiography," J. Am. Coll. Cardiol. 64(8), 772-780 (2014).

24. K. M. Chinnaiyan et al., "CT dose reduction using prospectively triggered or fast-pitch spiral technique employed in cardiothoracic imaging (the CT dose study)," J. Cardiovasc. Comput. Tomogr. 8(3), 205-214 (2014).

25. J. Hausleiter et al., "Image quality and radiation exposure with a low tube voltage protocol for coronary CT angiography results of the PROTECTION II trial," JACC Cardiovasc. Imaging 3(11), 1113-1123 (2010).

26. T. M. LaBounty et al., "Coronary CT angiography of patients with a normal body mass index using $80 \mathrm{kVp}$ versus $100 \mathrm{kVp}$ : a prospective, multicenter, multivendor randomized trial," Am. J. Roentgenol. 197(5), W860-W867 (2011). 
27. C. N. De Cecco et al., "Dual-source CT coronary angiography: prospective versus retrospective acquisition technique," Radiol. Med. (Torino) 116(2), 178-188 (2011).

28. L. Husmann et al., "Feasibility of low-dose coronary CT angiography: first experience with prospective ECG-gating," Eur. Heart J. 29(2), 191-197 (2008)

29. N. R. Mollet et al., "High-resolution spiral computed tomography coronary angiography in patients referred for diagnostic conventional coronary angiography," Circulation 112(15), 2318-2323 (2005).

30. "NCRP report no. 160, ionizing radiation exposure of the population of the United States," Bethesda, http://ncrponline.org/publications/reports/ ncrp-report-160/ (11 February 2017).

31. P. S. Douglas et al., "Outcomes of anatomical versus functional testing for coronary artery disease," N. Engl. J. Med. 372(14), 1291-1300 (2015).

32. J. M. Miller et al., "Diagnostic performance of coronary angiography by 64-row CT," N. Engl. J. Med. 359(22), 2324-2336 (2008).

33. SCOT-HEART Investigators, "CT coronary angiography in patients with suspected angina due to coronary heart disease (SCOT-HEART): an open-label, parallel-group, multicentre trial," The Lancet 385(9985), 2383-2391 (2015).

34. S. B. Puchner et al., "High-risk plaque detected on coronary CT angiography predicts acute coronary syndromes independent of significant stenosis in acute chest pain: results from the ROMICAT-II trial,' J. Am. Coll. Cardiol. 64(7), 684-692 (2014).

35. A. J. Taylor et al., "ACCF/SCCT/ACR/AHA/ASE/ASNC/NASCI/ SCAI/SCMR 2010 appropriate use criteria for cardiac computed tomography. A report of the American College of Cardiology Foundation Appropriate Use Criteria Task Force, the Society of Cardiovascular Computed Tomography, the American College of Radiology, the American Heart Association, the American Society of Echocardiography, the American Society of Nuclear Cardiology, the North American Society for Cardiovascular Imaging, the Society for Cardiovascular Angiography and Interventions, and the Society for Cardiovascular Magnetic Resonance," J. Am. Coll. Cardiol. 56(22), 1864-1894 (2010).

36. M. J. Budoff et al., "Diagnostic performance of 64-multidetector row coronary computed tomographic angiography for evaluation of coronary artery stenosis in individuals without known coronary artery disease: results from the prospective multicenter ACCURACY (assessment by coronary computed tomographic angiography of individuals undergoing invasive coronary angiography) trial," J. Am. Coll. Cardiol. 52(21), 1724-1732 (2008).

37. A. N. Primak et al., "Relationship between noise, dose, and pitch in cardiac multi-detector row CT," RadioGraphics 26(6), 1785-1794 (2006).

38. G. Sun et al., "320-detector row CT coronary angiography: effects of heart rate and heart rate variability on image quality, diagnostic accuracy and radiation exposure," Br. J. Radiol. 85(1016), e388-e394 (2012).

39. C.-M. Chen et al., "Radiation dose exposure of patients undergoing 320row cardiac CT for assessing coronary angiography and global left ventricular function," Int. J. Cardiovasc. Imaging 28(Suppl. 1), 1-5 (2012).

40. M. Y. Chen, S. M. Shanbhag, and A. E. Arai, "Submillisievert median radiation dose for coronary angiography with a second-generation 320-detector row CT scanner in 107 consecutive patients," Radiology 267(1), 76-85 (2013).

41. J. Qin et al., "320-detector CT coronary angiography with prospective and retrospective electrocardiogram gating in a single heartbeat: comparison of image quality and radiation dose," Br. J. Radiol. 85(1015), 945-951 (2012).

42. A. Padole et al., "CT radiation dose and iterative reconstruction techniques," Am. J. Roentgenol. 204(4), W384-W392 (2015).

43. D. E. Litmanovich et al., "Dose reduction in cardiothoracic CT: review of currently available methods," RadioGraphics 34(6), 1469-1489 (2014).

44. S. Oda et al., "A low tube voltage technique reduces the radiation dose at retrospective ECG-gated cardiac computed tomography for anatomical and functional analyses," Acad. Radiol. 18(8), 991-999 (2011).

45. F. Cademartiri and P. Pavone, "Advantages of retrospective ECG-gating in cardio-thoracic imaging with 16-row multislice computed tomography," Acta Bio-Med. 74(3), 126-130 (2003).

46. T. Techasith et al., "The effect of heart rhythm on patient radiation dose with dual-source cardiac computed tomography," J. Cardiovasc. Comput. Tomogr. 5(4), 255-263 (2011).

47. A. M. Lee et al., "Assessment of image quality and radiation dose of prospectively ECG-triggered adaptive dual-source coronary computed tomography angiography (cCTA) with arrhythmia rejection algorithm in systole versus diastole: a retrospective cohort study," Int. J. Cardiovasc. Imaging 29(6), 1361-1370 (2013).

48. A. M. Lee et al., "Coronary computed tomography angiography during arrhythmia: radiation dose reduction with prospectively ECG-triggered axial and retrospectively ECG-gated helical 128-slice dual-source CT,' J. Cardiovasc. Comput. Tomogr. 6(3), 172-183 (2012).

49. S. S. Halliburton et al., "SCCT guidelines on radiation dose and doseoptimization strategies in cardiovascular CT," J. Cardiovasc. Comput. Tomogr. 5(4), 198-224 (2011).

50. S. Leschka et al., "Scan length adjustment of CT coronary angiography using the calcium scoring scan: effect on radiation dose," Am. J. Roentgenol. 194(3), W272-W277 (2010).

51. M. K. Kalra et al., "Techniques and applications of automatic tube current modulation for CT," Radiology 233(3), 649-657 (2004).

52. T. Maruyama et al., "Radiation dose reduction and coronary assessability of prospective electrocardiogram-gated computed tomography coronary angiography: comparison with retrospective electrocardiogramgated helical scan," J. Am. Coll. Cardiol. 52(18), 1450-1455 (2008).

53. J. Menke et al., "Head-to-head comparison of prospectively triggered vs retrospectively gated coronary computed tomography angiography: meta-analysis of diagnostic accuracy, image quality, and radiation dose," Am. Heart J. 165(2), 154-163 (2013).

54. T. M. Labounty et al., "Effect of padding duration on radiation dose and image interpretation in prospectively ECG-triggered coronary CT angiography," Am. J. Roentgenol. 194(4), 933-937 (2010).

55. J. Hausleiter et al., "Radiation dose estimates from cardiac multislice computed tomography in daily practice: impact of different scanning protocols on effective dose estimates," Circulation 113(10), 1305-1310 (2006).

56. B. Bischoff et al., "Impact of a reduced tube voltage on CT angiography and radiation dose: results of the PROTECTION I study," JACC Cardiovasc. Imaging 2(8), 940-946 (2009).

57. W.-H. Yin et al., "Effect of reduced x-ray tube voltage, low iodine concentration contrast medium, and sinogram-affirmed iterative reconstruction on image quality and radiation dose at coronary CT angiography: results of the prospective multicenter REALISE trial," J. Cardiovasc. Comput. Tomogr. 9(3), 215-224 (2015).

58. T. A. Fuchs et al., "Coronary computed tomography angiography with model-based iterative reconstruction using a radiation exposure similar to chest X-ray examination," Eur. Heart J. 35(17), 1131-1136 (2014).

59. A. Schuhbaeck et al., "Image quality of ultra-low radiation exposure coronary CT angiography with an effective dose $<0.1 \mathrm{mSv}$ using high-pitch spiral acquisition and raw data-based iterative reconstruction," Eur. Radiol. 23(3), 597-606 (2013).

60. Y. Iyama et al., "Cardiac helical CT involving a low-radiation-dose protocol with a 100-kVp setting: usefulness of hybrid iterative reconstruction and display preset optimization," Medicine 95(46), e5459 (2016).

61. D. Andreini et al., "Coronary CT angiography with $80 \mathrm{kV}$ tube voltage and low iodine concentration contrast agent in patients with low body weight," J. Cardiovasc. Comput. Tomogr. 10(4), 322-326 (2016).

62. M. Meyer et al., "Closing in on the K edge: coronary CT angiography at 100,80 , and $70 \mathrm{kV}$-initial comparison of a second- versus a thirdgeneration dual-source CT system," Radiology 273(2), 373-382 (2014).

63. P. M. Linsen et al., "Computed tomography angiography with a 192-slice dual-source computed tomography system: improvements in image quality and radiation dose," J. Clin. Imaging Sci. 6(1), 44 (2016).

64. S. Mangold et al., "Coronary CT angiography in obese patients using 3rd generation dual-source CT: effect of body mass index on image quality," Eur. Radiol. 26(9), 2937-2946 (2016).

65. L. J. Zhang et al., "Image quality, radiation dose, and diagnostic accuracy of prospectively ECG-triggered high-pitch coronary CT angiography at $70 \mathrm{kVp}$ in a clinical setting: comparison with invasive coronary angiography," Eur. Radiol. 26(3), 797-806 (2016).

66. M. A. Bolen et al., "Image quality, contrast enhancement, and radiation dose of ECG-triggered high-pitch CT versus non-ECG-triggered standard-pitch CT of the thoracoabdominal aorta," Am. J. Roentgenol. 198(4), 931-938 (2012).

67. T. G. Flohr et al., "First performance evaluation of a dual-source CT (DSCT) system," Eur. Radiol. 16(2), 256-268 (2006). 
68. S. Achenbach et al., "Coronary computed tomography angiography with a consistent dose below $1 \mathrm{mSv}$ using prospectively electrocardiogram-triggered high-pitch spiral acquisition," Eur. Heart J. 31(3), 340-346 (2010).

69. B. B. Ghoshhajra et al., "Radiation dose reduction in pediatric cardiac computed tomography: experience from a Tertiary Medical Center," Pediatr. Cardiol. 35(1), 171-179 (2014).

70. T. Selcuk et al., "Effectiveness of using dual-source CT and the upshot it creates on both heart rate and image quality," Balk. Med. J. 33(3), 283-293 (2016).

71. P. Kröpil et al., "Prospectively ECG-triggered high-pitch spiral acquisition for cardiac CT angiography in routine clinical practice: initial results," J. Thorac. Imaging 27(3), 194-201 (2012).

72. L. Fu et al., "Comparison between pre-log and post-log statistical models in ultra-low-dose CT reconstruction," IEEE Trans. Med. Imaging 36, 707-720 (2016)

73. C. Naoum, P. Blanke, and J. Leipsic, "Iterative reconstruction in cardiac CT," J. Cardiovasc. Comput. Tomogr. 9(4), 255-263 (2015).

74. W.-H. Yin et al., "Iterative reconstruction to preserve image quality and diagnostic accuracy at reduced radiation dose in coronary CT angiography," JACC Cardiovasc. Imaging 6(12), 1239-1249 (2013).

75. L. Yang et al., "Optimization of hybrid iterative reconstruction level and evaluation of image quality and radiation dose for pediatric cardiac computed tomography angiography," Pediatr. Radiol. 47(1), 31-38 (2017).

76. P. Kröpil et al., "Impact of increasing levels of advanced iterative reconstruction on image quality in low-dose cardiac CT angiography," ROFO Fortschr. Geb. Rontgenstr. Nuklearmed. 186(6), 567-575 (2014).

77. L. L. Geyer et al., "State of the art: iterative CT reconstruction techniques," Radiology 276(2), 339-357 (2015).

78. A. J. Einstein et al., "Radiation dose from single-heartbeat coronary CT angiography performed with a 320-detector row volume scanner," Radiology 254(3), 698-706 (2010).

79. F. Khosa et al., "Influence of image acquisition on radiation dose and image quality: full versus narrow phase window acquisition using 320 MDCT," Sci. World J. 2013, e731590 (2013).

80. A. Prakash, A. J. Powell, and T. Geva, "Multimodality noninvasive imaging for assessment of congenital heart disease," Circ. Cardiovasc. Imaging 3(1), 112-125 (2010).

81. M. Koplay et al., "Prospective ECG-gated high-pitch dual-source cardiac CT angiography in the diagnosis of congenital cardiovascular abnormalities: radiation dose and diagnostic efficacy in a pediatric population," Diagn. Interventional Imaging 97(11), 1141-1150 (2016).

82. W. Gao et al., "Diagnostic accuracy of sub-mSv prospective ECGtriggering cardiac CT in young infant with complex congenital heart disease," Int. J. Cardiovasc. Imaging 32(6), 991-998 (2016).

83. dsct.editors@spiritlink.de, "Improvements in third generation dualsource CT and benefits for thoracic and cardiac CT in adult and pediatrics,"-Your Dual-source CT experts [Internet], http://www.dsct.com/ index.php/improvements-in-third-generation-dual-source-ct-and-benefitsfor-thoracic-and-cardiac-ct-in-adult-and-pediatrics/ (26 February 2017).

84. D. Andreini et al., "Low-dose CT coronary angiography with a novel IntraCycle motion-correction algorithm in patients with high heart rate or heart rate variability," Eur. Heart J. 16(10), 1093-1100 (2015).

85. H. Vadvala et al., "Coronary CTA using scout-based automated tube potential and current selection algorithm, with breast displacement results in lower radiation exposure in females compared to males," Cardiovasc. Diagn. Ther. 4(6), 470-479 (2014).

86. A. J. Einstein et al., "Effect of bismuth breast shielding on radiation dose and image quality in coronary CT angiography," J. Nucl. Cardiol. 19(1), 100-108 (2012).

87. P. M. Colletti, O. A. Micheli, and K. H. Lee, "To shield or not to shield: application of bismuth breast shields," Am. J. Roentgenol. 200(3), 503-507 (2013).

88. B. Bischoff et al., "Myocardial ischemia detection with single-phase CT perfusion in symptomatic patients using high-pitch helical image acquisition technique," Int. J. Cardiovasc. Imaging 33(4), 569-576 (2016).

89. A. So et al., "Technical note: evaluation of a $160-\mathrm{mm} / 256-$ row CT scanner for whole-heart quantitative myocardial perfusion imaging," Med. Phys. 43(8), 4821-4832 (2016).

90. I. Danad, B. Ó. Hartaigh, and J. K. Min, "Dual-energy computed tomography for detection of coronary artery disease," Expert Rev. Cardiovasc. Ther. 13(12), 1345-1356 (2015).

91. V. A. Murugan et al., "American College of Radiology Dose Index Registry: a user's guide for cardiothoracic radiologists part 1," J. Thorac. Imaging 30(6), W66-W68 (2015).

Biographies for the authors are not available. 